\title{
The Footprint of Diabetes Mellitus on the
}

\section{Characteristics and Response to Anti-Tuberculous Therapy in Patients with Pulmonary Tuberculosis from Saudi Arabia}

\author{
Rania Abd El-Hamid El-Kady ${ }^{1,2}$ \\ Safaa Abdulrahman Turkistani ${ }^{3}$ \\ 'Department of Medical Microbiology \\ and Immunology, Faculty of Medicine, \\ Mansoura University, Mansoura, Egypt; \\ ${ }^{2}$ Department of Pathological Sciences, \\ Fakeeh College for Medical Sciences, \\ Jeddah, Kingdom of Saudi Arabia; \\ ${ }^{3}$ Department of Medical Laboratory \\ Sciences, Fakeeh College for Medical \\ Sciences, Jeddah, Kingdom of Saudi \\ Arabia
}

Background: Right now, a tuberculosis (TB) and diabetes mellitus (DM) syndemic is reemerging worldwide. Given the contradictory results of the impact of DM on the natural history of pulmonary TB (PTB), this study was undertaken to shed light on the precision of this hypothesis from a community with a substantial caseload of both diseases.

Methods: The present 5-year, retrospective, cohort study involved 487 (60.8\% males, and $39.2 \%$ females) adult PTB patients (mean age 53.71 \pm 15.78 years) selected from Dr. Soliman Fakeeh Hospital (DSFH), Jeddah, Kingdom of Saudi Arabia (KSA). The relevant patients' clinical, radiological and microbiological data were extracted from the hospital medical and laboratory database.

Results: In our study, the cumulative prevalence of DM among PTB subjects was $27.1 \%$. Both diabetic and non-diabetic groups were matched with regard to gender $(p=0.46)$; however, diabetic patients were significantly older $(p=0.0001)$. Patients with concomitant DM displayed higher frequency of the classic clinical presentations of PTB and were 1.8 times more likely to have cavitary lesions on imaging studies $(p=0.012)$. Furthermore, diabetic patients showed higher initial sputum acid-fast bacillus (AFB) smear grade $(p=0.0001)$ and were more prone to have delayed culture conversion as compared to their non-diabetic counterparts $(77.55 \pm 37.74$ versus $54.95 \pm 27.67$ days, respectively; $p=0.0001$ ) which points out to less favorable treatment outcome. Conclusion: DM showed an impact on different aspects of PTB. Accordingly, integrated bidirectional screening programs for both diseases in the KSA need to be implemented to upgrade health-care services of patients with dual diagnosis.

Keywords: pulmonary, tuberculosis, diabetes, comorbidity, impact

\section{Introduction}

Despite 9 decades of effective vaccination campaigns and 6 decades of anti-tuberculous medications, tuberculosis (TB) remains the most common cause of death from a single infectious agent worldwide. ${ }^{1}$ Pulmonary tuberculosis (PTB) is an airborne communicable disease caused by Mycobacterium tuberculosis (MTB) bacilli, which are transmitted from person to person via inhalation of droplet nuclei classically generated by coughing. ${ }^{2}$ Recently, it is estimated that about $25 \%$ of the world's population have been infected with $M T B .{ }^{3}$ Unfortunately, COVID-19 pandemic was associated with a negative influence on TB control in terms of delayed diagnosis and increased severity of clinical manifestations of TB. ${ }^{4}$ 
In the Kingdom of Saudi Arabia (KSA), TB constitutes a public health concern owing to the population dynamics of the Kingdom, including an enormous number of emigrants from TB-endemic countries and the annual inflow of millions of hajjis during the Hajj and Umrah periods. ${ }^{5}$ Although a tangible advancement in the notification as well as the treatment of TB cases had occurred in Saudi Arabia, a reasonable infection rate is published, heretofore, as opposed to other countries. $^{6}$

In contrast to $\mathrm{TB}$, diabetes mellitus (DM), is a noncommunicable, metabolic disorder attributed to many factors. It is characterized by abnormally high blood glucose level secondary to disturbances in insulin secretion, insulin action or both. ${ }^{7}$ In 2019, DM was the fifth leading cause of global mortality, with approximately 463 million adults diagnosed with diabetes. By 2045, projection up to 548 million is anticipated. ${ }^{8}$

Historically, the correlation between DM and TB dates back to the beginning of the 1900s. Thereafter, this association was ignored due to the innovation of powerful treatment modalities for either disease. ${ }^{9}$ Globally, re-birth of this comorbidity was noticed because of current progress in the number of diabetic patients brought about by dramatic changes in the lifestyle. A meta-analysis observed that DM triples the possibility of developing active TB. ${ }^{10}$

The precise pathophysiology of DM as a detrimental factor for TB is not fully explored. It is believed that DM jeopardizes both specific and non-specific immune mechanisms. Diabetic patients have impaired chemotaxis, activation of phagocytic cells and presentation of the antigens of MTB bacilli. ${ }^{11}$ In addition, compromised $\mathrm{T}$ cellmediated immunity (CMI), decreased function of alveolar macrophages, reduced production of hydrogen peroxide and low levels of interferon- $\gamma$ are noticed. ${ }^{12}$

To the extent of our knowledge, few studies have been done in the Middle East countries to investigate the association between DM and TB. For that etiology, this study was designed to (i) assess the prevalence of DM in patients with PTB, (ii) review the impact of DM on the clinical, radiological and microbiological characteristics of patients diagnosed with PTB and (iii) evaluate the response of PTB cases with and without DM to anti-TB drugs, retrospectively, during a 5-year study period in patients attending Dr. Soliman Fakeeh Hospital (DSFH), Jeddah, KSA.

\section{Patients and Methods Study Eligibility, Design and Setting}

The study design was approved by the institutional review board (IRB) of DSFH (Approval No. 154/IRB/2020). In this retrospective cohort study, all confirmed adult ( $>18$ years) patients with active PTB attending DSFH and notified to the Saudi Ministry of Health (MOH) in the period from June 2015 to May 2020 were eligible to the study. DSFH is a 500-bedded private hospital in Jeddah, KSA. It provides both inpatient and outpatient tertiary-care for the residents of the Kingdom.

\section{Pulmonary TB Diagnostic Criteria}

During the study interval, 487 (296 males and 191 females) adult patients (mean age $53.71 \pm 15.78$ years) with newly diagnosed active PTB were identified based on their initial clinical presentation, radiologic findings as well as sputum acid-fast bacillus (AFB) smear results. The AFB smear microscopy was performed at the Microbiology Laboratory of DSFH using Ziehl-Neelsen (ZN) stain and smears were graded as,+++ , or +++ based on the criteria set by the International Union Against Tuberculosis and Lung Disease (IUATLD). ${ }^{13}$ Also, sputum specimens from the enrolled patients were cultured on Lowenstein-Jensen media in accordance with the standardized protocols of the hospital laboratory.

\section{Pulmonary TB Treatment Regimen}

Patients diagnosed with PTB were prescribed anti-TB medications according to the WHO recommendations. ${ }^{14}$ The patients were subjected to repeat sputum testing using Ziehl-Neelsen-stained smears as well as culture at the end of the initiation phase and after treatment completion.

\section{Diagnosis of DM Among the Enrolled Patients}

Patients with concurrent DM were identified based on the diagnostic scheme of the American Diabetes Association (ADA). ${ }^{15} \mathrm{DM}$ was considered poorly controlled if $\mathrm{HbA} 1 \mathrm{c}$ was $>8 \%$. ${ }^{16}$ The study cohort was further subdivided into 2 groups; group (1): patients suffering from both PTB and DM (DM/PTB group) and group (2): patients diagnosed with PTB only without DM (non-DM/PTB group).

\section{Patients' Demographic, Clinical and Radiologic Data}

Patients' medical record numbers (MRN) were used to capture the relevant data to the study cohort, including 
(1) demographic data; age at time of diagnosis, gender, nationality, occupation, (2) past history of PTB, (3) history of contact to PTB cases, (4) body mass index "BMI", (5) special habits including cigarette smoking and or alcohol consumption, (6) clinical presentation of PTB, (7) chest X-ray (CXR) and computed tomography (CT) findings, (8) results of the laboratory investigations and (9) response to anti-TB therapy based on sputum culture outcome.

\section{Exclusion Criteria}

Pediatric patients $(<18$ years), pregnant TB women and PTB patients with concomitant extrapulmonary TB, hepatitis B, hepatitis C, human immunodeficiency virus (HIV), autoimmune disease (s) or malignancy were excluded from the study. Also, patients receiving immunosuppressive therapy were not eligible to our study.

\section{Statistical Analysis}

The data were entered and analyzed using IBM $^{\circledR}$ SPSS $^{\circledR}$ Statistics program version 26.0 for Windows (SPSS Inc., Chicago, IL, USA). Categorical variables were expressed as numbers and percentages with Pearsons Chi-Square $\left(\chi^{2}\right)$ test used to define the statistical significance of the data, meanwhile Fischer's Exact test was used as a correction for $\chi^{2}$ test when $>25 \%$ of the cells have count $<5$ in $2 \times 2$ tables. Continuous variables were described as means \pm standard deviation (SD) for parametric data after testing normality using Kolmogorov-Smirnov test.

The Independent Samples T-Test was used to compare the means of 2 independent groups. Odds ratios (OR) with 95\% confidence intervals (CI) were determined. For sputum culture conversion time, a Kaplan-Meier plot was constructed and Log rank test was used for significance testing. A multivariate logistic regression analysis was performed to define the association of predictor variables with the outcome variable. $p$-values $<0.05$ (2-tailed) were considered statistically significant.

\section{Results}

\section{Demographic and Baseline} Characteristics of the Study Participants

From June 2015 to May 2020, 487 patients (60.8\% males and $39.2 \%$ females) with a mean age of $53.71 \pm 15.78$ years and a mean BMI of $24.37 \pm 3.70 \mathrm{~kg} / \mathrm{m}^{2}$ were eligible to the current study. About two-thirds of the enrolled patients were Saudi nationals. While about $45 \%$ of our cohort were self-employed, $5 \%$ and $4.7 \%$ were migrantworkers and college students, respectively.

Amongst our cohort, DM/PTB group (group 1) included 132 patients (27.1\%), whereas non-DM/PTB group (group 2) comprised 355 patients (72.9\%). A statistically significant difference was detected between both groups in relation to the age $(p=0.0001)$; however, they were matched with reference to gender $(p=0.46)$. At the time of PTB diagnosis, the BMI showed higher means $\pm \mathrm{SD}$ among comorbid patients compared to those without DM, with a statistically significant difference (Table 1).

\section{Glycemic Outlines of the Study Cohort}

Amongst $132 \mathrm{DM} / \mathrm{PTB}$ patients, the mean $\mathrm{HbA1c} \%$ was $10.58 \pm 2.33 \%$ compared to $5.09 \pm 0.56 \%$ in non-DM/PTB group $(p=0.0001)$. Other glycemic parameters are presented in Table 2. Almost $86 \%$ of our diabetic cohort had poor glycemic control based on $\mathrm{HbAlc} \%$, while $13.6 \%$ showed marginal HbA1c concentration (7.74 $\pm 0.16 \%)$.

Two-thirds of our patients had previous diagnosis of DM of 2.5-17 years' duration (mean $5.4 \pm 7.8$ years), meanwhile it was diagnosed in the other third during their follow-up visits in the hospital for PTB. About $89.4 \%$ of DM/PTB patients were on oral hypoglycemic pills and the remaining patients were scheduled insulin injections.

\section{Clinical Manifestations of Pulmonary TB Patients with and without DM}

Overall, productive cough was the most dominant clinical presentation of PTB in both DM/PTB and non-DM/PTB groups $(78.9 \%)$, followed by fever $(75.6 \%)$, night sweats $(65.7 \%)$ and unexplained weight loss (40\%). With the exception of chest pain and malaise, diabetic patients showed statistically significant differences in relation to the typical presentations of PTB as compared to nondiabetics (Table 3).

\section{Radiologic Findings of Pulmonary TB Patients According to Their DM Status}

On CXR and CT scan, patients having both comorbidities showed higher frequency of pulmonary consolidation as compared to those with PTB only (43.9\% versus $30.1 \%$ ), with a considerably significant difference (OR: 1.82, 95\% CI: $1.2-2.74, p=0.005)$. In the same way, pulmonary cavitary lesions were observed more commonly among diabetic patients (31.8\% versus 20.6\%, OR: 1.8, 95\% CI: 
Table I Baseline Characteristics of the Study Population

\begin{tabular}{|c|c|c|c|c|}
\hline Parameters & DM/PTB Group n = $132(\%)$ & Non-DM/PTB Group n = 355 (\%) & $\chi^{2}$ & $p$-value \\
\hline Age, years $( \pm S D)^{a}$ & $61.17 \pm 11.33$ & $50.94 \pm 16.3$ & 7.79 & $0.0001 *$ \\
\hline \multicolumn{5}{|l|}{ Gender } \\
\hline Males & $84(63.6 \%)$ & $212(59.7 \%)$ & 0.62 & 0.46 \\
\hline Females & 48 (36.4\%) & 143 (40.3\%) & & \\
\hline \multicolumn{5}{|l|}{ Nationality } \\
\hline Saudi & 85 (64.4\%) & $27 \mid(76.3 \%)$ & & \\
\hline Egyptian & $19(14.4 \%)$ & $33(9.3 \%)$ & & \\
\hline Filipino & 15 (II.4\%) & $29(8.2 \%)$ & 11.55 & 0.14 \\
\hline Others & $13(9.8 \%)$ & $22(6.2 \%)$ & & \\
\hline \multicolumn{5}{|l|}{ Occupation } \\
\hline Self-employed & $56(42.4 \%)$ & $162(45.6 \%)$ & & \\
\hline Un-employed & $48(36.4 \%)$ & I 32 (37.2\%) & 16.89 & 0.18 \\
\hline Business-owner & 21 (15.9\%) & $20(5.6 \%)$ & & \\
\hline Migrant-worker & $5(3.8 \%)$ & $20(5.6 \%)$ & & \\
\hline College student & $2(1.5 \%)$ & $21(5.9 \%)$ & & \\
\hline $\mathrm{BMI}, \mathrm{kg} / \mathrm{m}^{2}( \pm \mathrm{SD})^{\mathrm{a}}$ & $25.15 \pm 3.59$ & $24.08 \pm 3.71$ & 2.85 & $0.005^{*}$ \\
\hline Past history of PTB & $13(9.8 \%)$ & $24(6.8 \%)$ & 1.31 & 0.34 \\
\hline Contact to PTB case & 90 (68.2\%) & $215(60.6 \%)$ & 2.38 & 0.14 \\
\hline Cigarette smoking & $99(75 \%)$ & 250 (70.4\%) & 0.33 & 0.37 \\
\hline Alcohol drinking & 22 (16.7\%) & 43 (12.1\%) & 3.9 & 0.23 \\
\hline
\end{tabular}

Notes: ${ }^{a}$ Significance was tested using the independent samples $T$-test; ${ }^{*} p<0.05$ (statistically significant).

Abbreviations: DM/PTB, diabetes mellitus and pulmonary tuberculosis; $\mathrm{n}$, number; $\chi^{2}$, Pearsons Chi-Square test; SD, standard deviation; BMI, body mass index.

Table 2 Baseline Glycemic Parameters in Diabetic versus Non-Diabetic Pulmonary Tuberculosis Patients

\begin{tabular}{|l|c|c|c|c|}
\hline Parameters & DM/PTB Group $\mathbf{n}=132$ (\%) & Non-DM/PTB Group $\mathbf{n}=355$ (\%) & $\mathbf{9 5 \%} \mathbf{C l}$ & p-value \\
\hline FPG (mg/dL) & $210.59 \pm 42.69$ & $109.69 \pm 10.98$ & $96.08-105.73$ & $0.000 I^{*}$ \\
2-hours PPG (mg/dL) & $328.51 \pm 66.38$ & $180.8 \pm 17.19$ & $140.19-155.21$ & $0.000 I^{*}$ \\
HbAI c\% & $10.58 \pm 2.33 \%$ & $5.09 \pm 0.56 \%$ & $5.23-5.75$ & $0.000 I^{*}$ \\
\hline
\end{tabular}

Notes: ${ }^{*} p<0.05$ (statistically significant). Data are expressed as means \pm standard deviation (SD).

Abbreviations: DM/PTB, diabetes mellitus and pulmonary tuberculosis; $n$, number; $\mathrm{Cl}$, confidence interval; FPG, fasting plasma glucose; PPG, postprandial plasma glucose; HbAlc, glycosylated hemoglobin.

1.15-2.82, $p=0.012$ ). However, involvement of the lower lung lobes, pulmonary nodules as well as pleural effusion had more or less similar rates between both groups ( $p$-values $=0.16,0.54$ and 0.21 , respectively). Data are summarized in Table 3.

A subsequent multivariate logistic regression analysis revealed that age was not an independent risk factor for pulmonary cavitary lesions, meanwhile DM remained a significant predictor (OR: 2.01, 95\% CI: 1.24-3.24, $p=$ $0.004)$. On the other hand, additional multivariate analysis indicated that patients aged 30-44 years were 2.67 times more likely to have pulmonary consolidation $(p=0.001)$, which reflects a potential confounding effect of age. However, DM was also the most statistically significant factor (OR: 2.39, 95\% CI: 1.53-3.74, $p=0.0001$ ).

\section{Microbiological Profile of the Study Participants}

In our study, the proportion of patients with a preliminary positive AFB smear was statistically higher amongst the DM/PTB cohort as compared to the non-diabetics $(79.6 \%$ versus $60.8 \%$ ). In addition, 2 months after anti-TB therapy, 
Table 3 Clinical and Radiologic Findings of Pulmonary Tuberculosis Among Diabetic versus Non-Diabetic Patients

\begin{tabular}{|c|c|c|c|c|}
\hline Parameters & DM/PTB Group n = I32 (\%) & Non-DM/PTB Group n = 355 (\%) & OR $(95 \% \mathrm{Cl})$ & p-value \\
\hline \multicolumn{5}{|l|}{ Clinical findings } \\
\hline Cough & $116(87.9 \%)$ & $268(75.5 \%)$ & $2.35(1.32-4.19)$ & $0.004 *$ \\
\hline Fever & $110(83.3 \%)$ & $258(72.7 \%)$ & $1.88(1.13-3.14)$ & $0.01 *$ \\
\hline Night sweating & 97 (73.5\%) & $223(62.8 \%)$ & $1.64(1.05-2.55)$ & $0.03 *$ \\
\hline Dyspnea & $70(53 \%)$ & $96(27 \%)$ & $3.05(2.01-4.61)$ & $0.0001 *$ \\
\hline Weight loss & $68(51.5 \%)$ & $127(35.8 \%)$ & $1.9(1.27-2.86)$ & $0.002 *$ \\
\hline Anorexia & 59 (44.7\%) & $113(31.8 \%)$ & $1.73(1.15-2.61)$ & $0.01 *$ \\
\hline Malaise & $52(39.4 \%)$ & $112(31.5 \%)$ & $1.4(0.93-2.14)$ & 0.1 \\
\hline Chest pain & $43(32.6 \%)$ & $110(31 \%)$ & $1.08(0.70-1.65)$ & 0.74 \\
\hline Hemoptysis & $36(27.3 \%)$ & $63(17.7 \%)$ & $1.74(1.08-2.78)$ & $0.02 *$ \\
\hline \multicolumn{5}{|c|}{ Pulmonary radiologic findings } \\
\hline Consolidation & $58(43.9 \%)$ & $107(30.1 \%)$ & $1.82(1.2-2.74)$ & $0.005^{*}$ \\
\hline Cavitation & 42 (31.8\%) & $73(20.6 \%)$ & I.8 (1.15-2.82) & $0.012 *$ \\
\hline Lower lung lesions & $26(19.7 \%)$ & $5 \mathrm{I}(\mathrm{I} 4.4 \%)$ & $1.46(0.87-2.46)$ & 0.16 \\
\hline Nodules & $20(15.2 \%)$ & 45 (12.7\%) & $1.23(0.69-2.17)$ & 0.54 \\
\hline Pleural effusion & $13(9.8 \%)$ & $23(6.5 \%)$ & $1.6(0.77-3.21)$ & 0.21 \\
\hline
\end{tabular}

Notes: $*_{p}<0.05$ (statistically significant). Data are expressed as numbers and percentages.

Abbreviations: DM/PTB, diabetes mellitus and pulmonary tuberculosis; $n$, number; OR, odds ratio; $\mathrm{Cl}$, confidence interval.

negative sputum smears were more probably to be detected among non-DM/PTB group in contrast to diabetics $(67.6 \%$ versus $43.9 \%$, respectively). Details are illustrated in Table 4.

Notably, $57.6 \%$ of the diabetic patients had their sputum culture converted to negative after 2 months of anti-TB therapy, in comparison to $77.7 \%$ of the nondiabetics $(p=0.0001)$. Kaplan-Meier curves revealed a considerably delayed sputum culture conversion to negative $\left(\chi^{2}=43.14, p=0.0001\right)$ among diabetic as compared to non-diabetic patients (mean time; $77.55 \pm$ 37.74 and $54.95 \pm 27.67$ days, respectively). Data are shown in Figure 1.
To assess the effect of further confounders as predictors for sputum culture conversion, we generated a multivariate regression analysis. Intriguingly, DM was the most statistically significant predictor for delayed sputum culture conversion $(p=0.001)$, with diabetics were 2.28 times more likely to experience delayed culture conversion (Table 5). However, 45-60 years of age had a confounding effect on the treatment response of PTB $(p=0.01)$.

\section{Discussion}

To date, TB and DM comorbidity represent a serious public health challenge despite deliberate efforts to decrease the burden of either disease. Though numerous prospective

Table 4 Sputum Acid-Fast Bacillus (AFB) Smear Results in This Study

\begin{tabular}{|c|c|c|c|c|}
\hline Parameters & DM/PTB Group n = I32 (\%) & Non-DM/PTB Group n = 355 (\%) & $\chi^{2}$ & p-value \\
\hline \multicolumn{5}{|c|}{ Baseline sputum smear results } \\
\hline Negative & 27 (20.5\%) & 139 (39.2\%) & \multirow{3}{*}{$18.7 \mid$} & \multirow{3}{*}{$0.0001 *$} \\
\hline Grade ++ & $69(52.3 \%)$ & $163(45.9 \%)$ & & \\
\hline Grade +++ & $36(27.3 \%)$ & $53(14.9 \%)$ & & \\
\hline \multicolumn{5}{|c|}{ Two months' post-treatment smear results } \\
\hline Negative & 58 (43.9\%) & 240 (67.6\%) & \multirow{3}{*}{43.32} & \multirow{3}{*}{$0.0001 *$} \\
\hline Grade + & $48(36.4 \%)$ & $103(29.0 \%)$ & & \\
\hline Grade ++ & 26 (19.7\%) & 12 (3.4\%) & & \\
\hline
\end{tabular}

Notes: $*_{p}<0.05$ (statistically significant). Data are expressed as numbers and percentages.

Abbreviations: DM/PTB, diabetes mellitus and pulmonary tuberculosis; $n$, number; $\chi^{2}$, Pearsons Chi-Square test. 


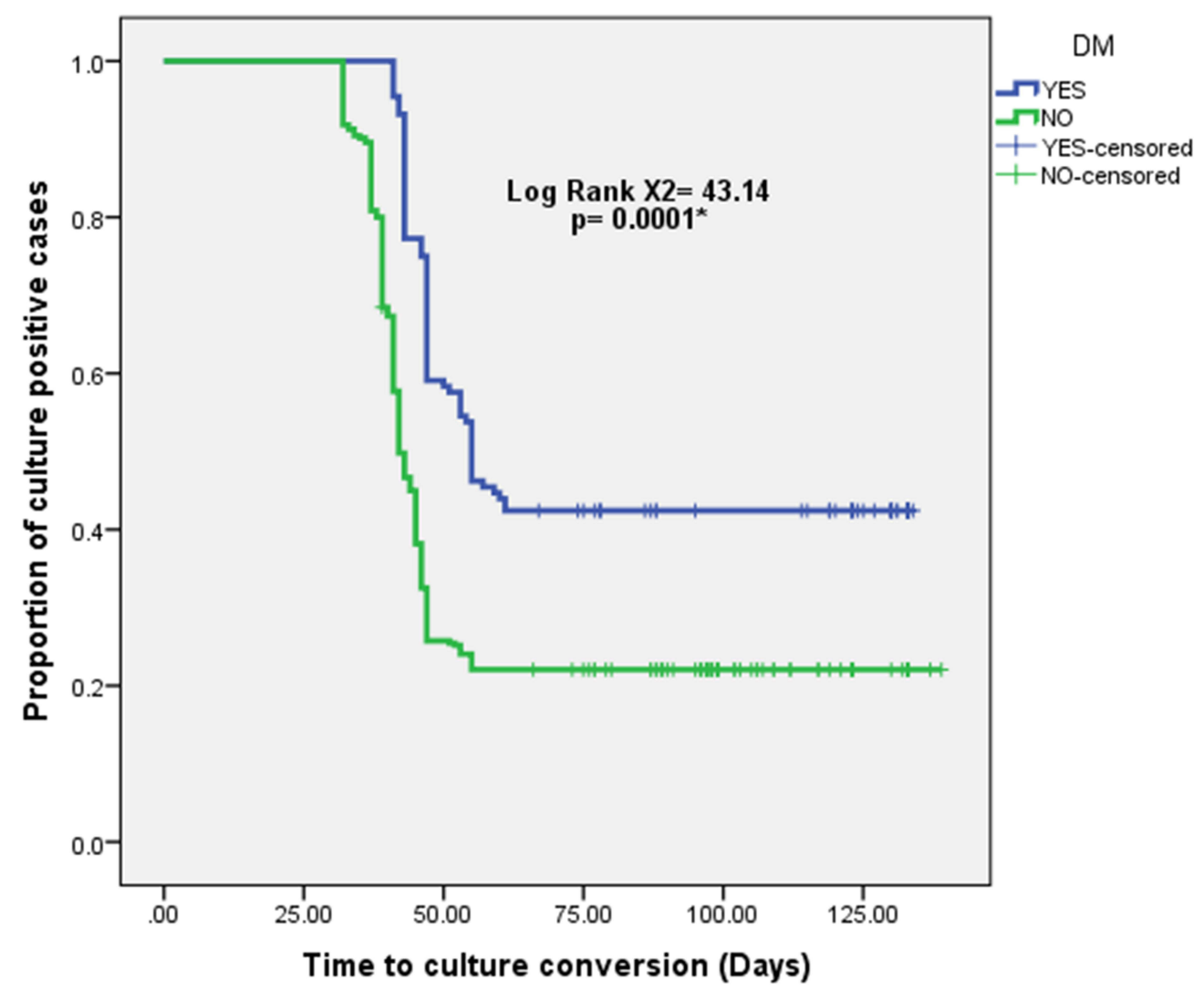

Figure I Kaplan-Meier plot of the time to sputum culture conversion after treatment initiation among pulmonary TB (PTB) patients with and without diabetes mellitus (DM). Kaplan-Meier curves revealed significantly longer sputum time to culture conversion into negative among DM/PTB patients (mean time: $77.55 \pm 37.74$ days) as compared to their non-diabetic counterparts (mean time: $54.95 \pm 27.67$ days). Significance was tested using the Log rank test $\left(\chi^{2}=43.14, p=0.000 \mathrm{I}\right.$ ). Of note, $57.6 \%$ of the diabetic cohort had their sputum culture converted to negative after 2 months of anti-TB therapy, in contrast to $77.7 \%$ of the non-diabetics. $* p<0.05$ (statistically significant).

and retrospective studies have investigated the correlation between both conditions, currently available data from the $\mathrm{KSA}$, in this perspective, are limited. With this in mind, we performed this 5 -year retrospective study.

Overall, 487 patients diagnosed with PTB during the study interval were found to fulfill our inclusion criteria. DM was identified in $27.1 \%$ of the study group. This prevalence is supported by the findings of a recent study performed in King Khalid University Hospital, Riyadh, Saudi Arabia, where DM was detected in $26.7 \%$ of their TB cohort. ${ }^{17}$ This high prevalence of DM among PTB patients from the KSA warrants prompt efforts from the policymakers. Parallel to that, a prevalence rate of $27.7 \%$ has been declared from analogous study conducted in Egypt. ${ }^{18}$ Likewise, an overall prevalence of $25.3 \%$ has been reported from another study conducted in India, ${ }^{19}$ which is the head TB-burden country all over the world. ${ }^{20}$

Recently, a South Asian systematic review concluded that DM accounted for $21 \%$ among the enrolled TB patients. The investigators acclaimed this rate to the fact that most of the Asians suffer from adiposity, poor glycemic control, reduced functional pancreatic $\beta$-cell mass as well as decreased insulin secretion. ${ }^{21}$ On the other hand, a Brazilian study noticed that the mean prevalence of TB/ DM comorbidity during the study period was $11.29 \% .{ }^{22}$ Dissimilar results could be explained by dissimilar ethnic, economic and epidemiological characteristics among different nations.

Interestingly, a prevalence about $3 \%$ was disclosed from an earlier study performed in Mozambique. Furthermore, an estimated prevalence of $1 \%$ was observed in Beira, the second largest city in Mozambique. This result was attributed to the lack of some of the risk factors for DM such as obesity, old age ( $>45$ years), hypertension and smoking. ${ }^{23}$ In light of that, Di Gennaro and his colleagues recommended DM screening in resource-poor countries, including the aforementioned ones, using a single blood sugar testing irrespective of other factors like education, social background and availability of qualified health-care workers. ${ }^{24}$ 
In the present work, patients with co-occurring DM and PTB tended to be significantly older in age compared to those with PTB only $(p=0.0001)$. This variance could be related to the possibility of PTB reactivation in elderly patients along with increased incidence of DM among this age group. Our finding is in keeping with the results from a prior nationwide cohort study. In that retrospective study, Gil-Santana and his associates noticed that $\mathrm{TB} / \mathrm{DM}$ patients were considerably older $(p<0.001)$ than non-diabetics enrolled at a TB primary care facility in Brazil between 2004 and 2010. ${ }^{25}$ Conversely, an earlier Iranian study found no significant age difference amongst the recruited group. ${ }^{26}$

When stratified with gender, a male preponderance was noted in both diabetic and non-diabetic subjects; however, the difference did not reach a statistical significance $(p=$ 0.46). Our result is concordant with the observation of a cross-sectional study from Pakistan. ${ }^{27}$ This finding could partly be explained by differences in lifestyle, as we noticed a higher rate of cigarette smoking and alcohol consumption, in the present study, among males compared to the females ( $p$-values $=0.01$ and 0.006 , respectively) which are both risk factors for PTB. ${ }^{28}$

In favor of our results, a Brazilian study disclosed that diabetic individuals were more likely to have classic clinical manifestations of PTB. ${ }^{29}$ Additionally, input from a Chinese study deduced that DM was independently related to worse chest symptoms in PTB patients. ${ }^{30}$ This could be traced to the deranged innate and adaptive immune mechanisms in patients with coincident DM. Consequently, failure to eliminate $M T B$ bacilli enables bacterial multiplication and dissemination to occur more rapidly, contributing to aggravated clinical features in hyperglycemic patients. ${ }^{31}$ Given the fact that most of the herein diabetic patients $(86 \%)$ had $\mathrm{HbAlc}>8 \%$ (poorly controlled DM) and the remaining subjects had borderline mean $\mathrm{HbA} 1 \mathrm{c} \%$ of $7.74 \pm 0.16 \%$, we were not able to conduct a sub-group analysis of our DM cohort according to their degree of dysglycemia.

Compatible with our results, previous studies have also explored conceivable effects of DM on the radiologic findings of PTB. An earlier study conducted in Riyadh, KSA and another one from Bangladesh endorsed that diabetic patients had pulmonary cavities more often than non-diabetics $(p \text {-values }=0.02 \text { and } 0.001 \text {, respectively) })^{32,33}$ This could be ascribed to the immune dysfunction in diabetic patients induced by hyperglycemia. ${ }^{34}$

Up till now, treatment response in $\mathrm{DM} / \mathrm{PTB}$ patients is questionable. Sputum AFB smear and culture negativity are key leaders of treatment outcome after commencing anti-TB therapy. In our study, patients with concurrent DM showed a higher initial sputum smear grade (which is the milestone of MTB bacilli density) as compared to nondiabetics. Also, 2 months after anti-TB therapy, nondiabetic patients were more likely to have negative sputum smears (Table 4). Observations of this study are consistent with the findings of previous authors. ${ }^{35-37}$ Quite the reverse, an earlier case-control study contradicted this result. ${ }^{38}$ That incompatible conclusion could be explained by differences in the duration of their study, demographics of the study participants, baseline MTB load as well as their relatively small sample size.

More importantly, the lag time till conversion of sputum cultures to negative during anti-TB therapy is used for forecasting treatment response. Of the total $132 \mathrm{DM} / \mathrm{PTB}$ patients, $76(57.6 \%)$ got their sputum culture converted to negative, in contrast to $276(77.7 \%)$ non-diabetic patients $(p=0.0001)$, with the mean duration for sputum culture conversion to negative was significantly longer in DM/ PTB patients compared to non-DM/PTB group (details are given in Figure 1). In the multivariate logistic regression analysis, DM remained the most significant predictor for delayed sputum culture conversion (Table 5).

In agreement with our findings, a study from Georgia and another one from Qatar ascertained unfavorable outcome of sputum culture conversion amongst diabetics. ${ }^{39,40}$ On the other side, a recent prospective study from India affirmed that DM has no adverse impact on sputum culture results. Nevertheless, the follow-up period in that study was only 18 months which could contribute to this discordance. ${ }^{41}$ Many investigators have endeavored to provide clarifications for delayed sputum culture conversion among diabetic subjects. For example, Lyu and his co-workers acknowledged that long-term hyperglycemia decreases the secretion of gastric hydrochloric acid, which adversely interferes with the pharmacokinetic parameters of rifampicin. ${ }^{42}$ Furthermore, other authors suggested that diabetic gastroparesis could partly contribute to impaired gastrointestinal absorption of anti-TB medications. ${ }^{43}$

In addition to that, our multivariate analysis indicated that 45-60 years of age had a confounding effect on treatment outcome of PTB $(p=0.01)$. Lately, a study from Italy addressed factors contributing to adverse events (AEs) and poor treatment outcome of PTB. The research group found that aging and DM are amongst the predictors of AEs for PTB. ${ }^{44}$ Accordingly, frequent follow-up of 
Table 5 Multivariate Logistic Regression Analysis of the Predictors for Sputum Culture Conversion in This Study

\begin{tabular}{|c|c|c|c|c|c|}
\hline \multirow[t]{2}{*}{ Parameters } & \multirow[t]{2}{*}{ Culture Conversion } & \multirow[t]{2}{*}{ No Culture Conversion } & \multirow[t]{2}{*}{ Univariate $p$-value } & \multicolumn{2}{|c|}{ Multivariate } \\
\hline & & & & OR (95\% Cl) & $p$-value \\
\hline \multicolumn{6}{|l|}{ Age group } \\
\hline$<30$ years & $25(7.1 \%)$ & $6(4.4 \%)$ & & $0.54(0.2-1.42)$ & 0.21 \\
\hline 30-44 years & 87 (24.7\%) & $22(16.3 \%)$ & $0.007^{*}$ & $0.55(0.31-0.98)$ & 0.45 \\
\hline $45-60$ years & 121 (34.4\%) & 39 (28.9\%) & & $0.53(0.33-0.87)$ & $0.01^{*}$ \\
\hline$>60$ years & $119(33.8 \%)$ & $68(50.4 \%)$ & & - & - \\
\hline \multicolumn{6}{|l|}{ Gender } \\
\hline Male & $219(62.2 \%)$ & 77 (57\%) & 0.3 & $0.72(0.47-1.09)$ & 0.12 \\
\hline Female & I 33 (37.8\%) & $58(43 \%)$ & & & \\
\hline \multicolumn{6}{|l|}{ DM } \\
\hline Yes & $76(21.6 \%)$ & $56(4 \mid .5 \%)$ & $0.0001 *$ & $2.28(1.39-3.72)$ & $0.001 *$ \\
\hline No & $276(78.4 \%)$ & 79 (58.5\%) & & & \\
\hline \multicolumn{6}{|c|}{ Sputum smear grade (Baseline) } \\
\hline Negative & $122(34.7 \%)$ & $44(32.6 \%)$ & & $\mathrm{I} .02(0.56-1.87)$ & 0.95 \\
\hline++ & $168(47.7 \%)$ & $64(47.4 \%)$ & 0.81 & $0.98(0.56-1.73)$ & 0.95 \\
\hline+++ & $62(17.6 \%)$ & $27(20 \%)$ & & - & - \\
\hline \multicolumn{6}{|c|}{ Sputum smear grade ( 2 months after treatment) } \\
\hline Negative & $228(64.8 \%)$ & 70 (51.9\%) & & $0.93(0.43-2.02)$ & 0.85 \\
\hline+ & 99 (28.1\%) & 52 (38.5\%) & $0.03 *$ & $1.47(0.67-3.26)$ & 0.34 \\
\hline++ & $25(7.1 \%)$ & $13(9.6 \%)$ & & - & - \\
\hline
\end{tabular}

Note: $*_{p}<0.05$ (statistically significant).

Abbreviations: DM, diabetes mellitus; OR, odds ratio; $\mathrm{Cl}$, confidence interval.

those patients by the health-care professionals is of utmost importance. Currently, there is a growing interest for the potential use of chest ultrasound (CUS) to diagnose and monitor PTB cases. Di Gennaro and others concluded that CUS is a promising imaging modality for recognition of TB-related conditions. ${ }^{45}$ Given the availability and safety issues, CUS should be employed in different health-care facilities for optimum follow-up of PTB patients, especially those with likely AEs.

With regard to the prevalence of multidrug-resistant (MDR)-MTB in Saudi Arabia, 4\% of the total isolates identified in the central tuberculosis laboratory in AlMadinah Al-Monawarah in the period from January 2012 to December 2014 were MDR. ${ }^{46}$ This high rate could be related to the unique nature of Al-Madinah, where millions of Muslims including those from TB-endemic regions frequently visit this holy place, especially during Hajj and Umrah seasons. Another study from Tabuk province, north of the KSA, demonstrated that $3.3 \%$ of the isolates were MDR. ${ }^{47}$ Unfortunately, susceptibility testing for MTB isolates was not routinely done at the Microbiology Laboratory of DSFH during the study interval.
Accordingly, we were not able to gauge the extent of this problem.

Of note, our study has some limitations that worthmentioning. First, the retrospective nature of our study makes it liable to selection bias of the study cohort. Second, cultural characteristics of the Saudi society may force some of the enrolled patients to deny smoking or alcohol drinking, which could modify our results related to behavioral risk factors for coincident DM and PTB. Finally, we were not able to figure out susceptibility pattern of MTB isolates to anti-TB drugs because of the shortage of data from our Microbiology laboratory records in this regard.

\section{Conclusion}

Generally, our tertiary-care facility showed a high prevalence of PTB/DM comorbidity. In addition, DM had an unpromising effect on the clinical, radiological and microbiological profile of PTB subjects as well as their treatment outcome from a country with a considerable concern of either disease. This underscores the need to reciprocal screening for PTB and DM, which could act as a gateway in timely detection and management of whichever disease. 
Also, public awareness regarding truths and myths of PTB and DM should be underlined on a large scale in different communities regardless of their educational level. Finally, it is mandatory to manage all $\mathrm{TB}$ patients taking into account their increased risk of non-communicable diseases, including DM.

\section{Data Sharing Statement}

The data used to support the findings of this study are available from the corresponding author upon request.

\section{Ethics Approval and Informed Consent}

The study was conducted in accordance with the Declaration of Helsinki. The study protocol was approved by the institutional review board (IRB) of DSFH (Approval No. 154/IRB/2020) and the need for informed consent was waived since all data were anonymized before analysis. Patients' data privacy and confidentiality were respected in all levels of the study.

\section{Funding}

There is no funding to report.

\section{Disclosure}

The authors have no conflicts of interest for this work to declare.

\section{References}

1. Silva DR, Mello FCQ, Migliori GB. Tuberculosis series 2020. J Bras Pneumol. 2020;46(2):e20200027. doi:10.36416/1806-3756/e20200027

2. Tellier R, Li Y, Cowling BJ, Tang JW. Recognition of aerosol transmission of infectious agents: a commentary. BMC Infect Dis. 2019;19 (1):101. doi:10.1186/s12879-019-3707-y

3. Koegelenberg CFN, Schoch OD, Lange C. Tuberculosis: the past, the present and the future. Respiration. 2021;100(7):553-556. doi:10.1159/000516509

4. Di Gennaro F, Gualano G, Timelli L, et al. Increase in Tuberculosis Diagnostic Delay during First Wave of the COVID-19 Pandemic: data from an Italian Infectious Disease Referral Hospital. Antibiotics. 2021;10(3):272. doi:10.3390/antibiotics 10030272

5. Almutairi FM, Tayeb T, Alhakeem R, Saeed AB, Assiri A, McNabb SJN. Distribution and determinants of tuberculosis in the Kingdom of Saudi Arabia from 2005 to 2012. J Epidemiol Glob Health. 2018;Suppl(Suppl 1):S23-S28. doi:10.1016/j.jegh.2017.08.002

6. Al-Sahafi A, Al-Sayali MM, Mandoura N, et al. Treatment outcomes among tuberculosis patients in Jeddah, Saudi Arabia: results of a community mobile outreach directly observed Treatment, Short-course (DOTS) project, compared to a standard facility-based DOTS: a randomized controlled trial. J Clin Tuberc Other Mycobact Dis. 2020;22:100210. doi:10.1016/j.jctube.2020.100210

7. Peer N, Balakrishna Y, Durao S. Screening for type 2 diabetes mellitus. Cochrane Database Syst Rev. 2020;5(5):CD005266. doi:10.1002/14651858.CD005266
8. Wu Y, Fu R, Lei C, et al. Estimates of Type 2 Diabetes Mellitus Burden Attributable to Particulate Matter Pollution and Its 30-Year Change Patterns: a Systematic Analysis of Data From the Global Burden of Disease Study 2019. Front Endocrinol. 2021;12:689079. doi:10.3389/fendo.2021.689079

9. van Crevel R, Critchley JA. The interaction of diabetes and tuberculosis: translating research to policy and practice. Trop Med Infect Dis. 2021;6(1):8. doi:10.3390/tropicalmed6010008

10. Chen Z, Liu Q, Song R, et al. The association of glycemic level and prevalence of tuberculosis: a meta-analysis. BMC Endocr Disord. 2021;21(1):123. doi:10.1186/s12902-021-00779-6

11. Solá E, Rivera C, Mangual M, Martinez J, Rivera K, Fernandez R. Diabetes mellitus: an important risk factor for reactivation of tuberculosis. Endocrinol Diabetes Metab Case Rep. 2016;2016:16-0035. doi:10.1530/EDM-16-0035

12. Ayelign B, Negash M, Genetu M, Wondmagegn T, Shibabaw T. Immunological impacts of diabetes on the susceptibility of Mycobacterium tuberculosis. J Immunol Res. 2019;2019:6196532. doi:10.1155/2019/6196532

13. International Union Against Tuberculosis and Lung Disease (IUATLD). Technical Guide. Sputum Examination for Tuberculosis by Direct Microscopy in Low Income Countries. 5th ed. 2000.

14. World Health Organization. Treatment of Tuberculosis: Guidelines. 4th ed. Geneva, Switzerland: World Health Organization; 2010.

15. American Diabetes Association. Standards of medical care in diabetes-2019 abridged for primary care providers. Clin Diabetes. 2019;37(1):11-34. doi:10.2337/cd18-0105

16. Hewage S, Somasundaram N, Ratnasamy V, et al. Active screening of patients with diabetes mellitus for pulmonary tuberculosis in a tertiary care hospital in Sri Lanka. PLoS One. 2021;16(4): e0249787. doi:10.1371/journal.pone.0249787

17. Binkhamis KM, Bahatheg MA, Altahan FA, et al. Prevalence and outcome of isoniazid-monoresistant tuberculosis at a university hospital in Saudi Arabia. Saudi Med J. 2021;42(6):636-642. doi:10.15537/smj.2021.42.6.20200832

18. Noubiap JJ, Nansseu JR, Nyaga UF, et al. Global prevalence of diabetes in active tuberculosis: a systematic review and metaanalysis of data from $2 \cdot 3$ million patients with tuberculosis. Lancet Glob Health. 2019;7(4):e448-e460. doi:10.1016/S2214-109X(18) 30487-X

19. Pande T, Huddart S, Xavier W, et al. Prevalence of diabetes mellitus amongst hospitalized tuberculosis patients at an Indian tertiary care center: a descriptive analysis. PLoS One. 2018;13(7):e0200838. doi:10.1371/journal.pone. 0200838

20. Dabhi PA, Thangakunam B, Gupta R, et al. Screening for prevalence of current TB disease and latent TB infection in type 2 diabetes mellitus patients attending a diabetic clinic in an Indian tertiary care hospital. PLoS One. 2020;15(6):e0233385. doi:10.1371/journal. pone. 0233385

21. Gautam S, Shrestha N, Mahato S, Nguyen TPA, Mishra SR, BergBeckhoff G. Diabetes among tuberculosis patients and its impact on tuberculosis treatment in South Asia: a systematic review and meta-analysis. Sci Rep. 2021;11(1):2113. doi:10.1038/s41598-02181057-2

22. Sousa GGDS, Pascoal LM, Costa ACPJ, et al. Trend and factors associated with tuberculosis-diabetes mellitus comorbidity in a northeastern Brazilian municipality. Rev Bras Enferm. 2021;74(3): e20201238. doi:10.1590/0034-7167-2020-1238

23. Pizzol D, Di Gennaro F, Chhaganlal KD, et al. Prevalence of diabetes mellitus in newly diagnosed pulmonary tuberculosis in Beira, Mozambique. Afr Health Sci. 2017;17(3):773-779. doi:10.4314/ahs. v17i3.20

24. Di Gennaro F, Marotta C, Antunes M, Pizzol D. Diabetes in active tuberculosis in low-income countries: to test or to take care? Lancet Glob Health. 2019;7(6):e707. doi:10.1016/S2214-109X(19)30173-1 
25. Cordeiro da Costa J, Oliveira O, Baía L, Gaio R, Correia-Neves M, Duarte R. Prevalence and factors associated with diabetes mellitus among tuberculosis patients: a nationwide cohort. Eur Respir J. 2016;48(1):264-268. doi:10.1183/13993003.00254-2016

26. Alavi SM, Khoshkho MM, Salmanzadeh S, Eghtesad M. Comparison of epidemiological, clinical, laboratory and radiological features of hospitalized diabetic and non-diabetic patients with pulmonary tuberculosis at razi hospital in ahvaz. Jundishapur J Microbiol. 2014;7(9): e12447. doi: $10.5812 /$ jjm. 12447

27. Tahir Z, Ahmad MU, Akhtar AM, Yaqub T, Mushtaq MH, Javed H. Diabetes mellitus among tuberculosis patients: a cross sectional study from Pakistan. Afr Health Sci. 2016;16(3):671-676. doi:10.4314/ahs. v16i3.5

28. Wessels J, Walsh CM, Nel M. Smoking habits and alcohol use of patients with tuberculosis at Standerton Tuberculosis Specialised Hospital, Mpumalanga, South Africa. Health SA. 2019;24:1146. doi:10.4102/hsag.v24i0.1146

29. Gil-Santana L, Almeida-Junior JL, Oliveira CA, et al. Diabetes is associated with worse clinical presentation in tuberculosis patients from Brazil: a Retrospective Cohort Study. PLoS One. 2016;11(1): e0146876. doi:10.1371/journal.pone.0146876

30. Leung CC, Yew WW, Mok TYW, et al. Effects of diabetes mellitus on the clinical presentation and treatment response in tuberculosis. Respirology. 2017;22(6):1225-1232. doi:10.1111/resp.13017

31. Kumar Nathella P, Babu S. Influence of diabetes mellitus on immunity to human tuberculosis. Immunology. 2017;152(1):13-24. doi:10.1111/imm.12762

32. Alkabab YM, Enani MA, Indarkiri NY, Heysell SK. Performance of computed tomography versus chest radiography in patients with pulmonary tuberculosis with and without diabetes at a tertiary hospital in Riyadh, Saudi Arabia. Infect Drug Resist. 2018;11:37-43. doi:10.2147/IDR.S151844

33. Paul KK, Alkabab YMA, Rahman MM, et al. A public-private model to scale up diabetes mellitus screening among people accessing tuberculosis diagnostics in Dhaka, Bangladesh. Int $J$ Infect Dis. 2020;92:56-61. doi:10.1016/j.ijid.2020.01.001

34. Huang LK, Jiang LD, Lai YC, Wu MH, Chang SC. Pulmonary tuberculous cavities in diabetic patients: glycemic control is still the dominant factor despite the emerging role of metformin. J Chin Med Assoc. 2019;82(8):628-634. doi:10.1097/JCMA

35. Asemahagn MA. Sputum smear conversion and associated factors among smear-positive pulmonary tuberculosis patients in East Gojjam Zone, Northwest Ethiopia: a longitudinal study. BMC Pulm Med. 2021;21(1):118. doi:10.1186/s12890-021-01483-w

36. Shariff NM, Safian N. Diabetes mellitus and its influence on sputum smear positivity at the 2nd month of treatment among pulmonary tuberculosis patients in Kuala Lumpur, Malaysia: a case control study. Int $J$ Mycobacteriol. 2015;4(4):323-329. doi:10.1016/j. ijmyco.2015.09.003
37. Viswanathan AA, Gawde NC. Effect of type II diabetes mellitus on treatment outcomes of tuberculosis. Lung India. 2014;31(3):244-248. doi:10.4103/0970-2113.135764

38. Singla R, Bharty SK, Gupta UA, et al. Sputum smear positivity at two months in previously untreated pulmonary tuberculosis patients. Int J Mycobacteriol. 2013;2(4):199-205. doi:10.1016/j.ijmyco.2013.0 8.002

39. Salindri AD, Kipiani M, Kempker RR, et al. Diabetes Reduces the Rate of Sputum Culture Conversion in Patients With Newly Diagnosed Multidrug-Resistant Tuberculosis. Open Forum Infect Dis. 2016;3(3):ofw126. doi:10.1093/ofid/ofw126

40. Dousa KM, Hamad A, Albirair M, et al. Impact of diabetes mellitus on the presentation and response to treatment of adults with pulmonary tuberculosis in qatar. Open Forum Infect Dis. 2018;6(1):ofy335. doi:10.1093/ofid/ofy335

41. Mave V, Gaikwad S, Barthwal M, et al. Diabetes Mellitus and Tuberculosis Treatment Outcomes in Pune, India. Open Forum Infect Dis. 2021;8(4):ofab097. doi:10.1093/ofid/ofab097

42. Lyu M, Wang D, Zhao J, et al. A novel risk factor for predicting anti-tuberculosis drug resistance in patients with tuberculosis complicated with type 2 diabetes mellitus. Int $J$ Infect Dis. 2020;97:69-77. doi:10.1016/j.ijid.2020.05.080

43. Ghimire S, Karki S, Maharjan B, et al. Treatment outcomes of patients with MDR-TB in Nepal on a current programmatic standardised regimen: retrospective single-centre study. BMJ Open Respir Res. 2020;7(1):e000606. doi:10.1136/bmjresp-2020-000606

44. Di Gennaro F, Vittozzi P, Gualano G, et al. Active Pulmonary Tuberculosis in Elderly Patients: a 2016-2019 Retrospective Analysis from an Italian Referral Hospital. Antibiotics. 2020;9 (8):489. doi:10.3390/antibiotics9080489

45. Di Gennaro F, Pisani L, Veronese N, et al. Potential diagnostic properties of chest ultrasound in thoracic tuberculosis-a systematic review. Int $J$ Environ Res Public Health. 2018;15(10):2235. doi:10.3390/ijerph15102235

46. Elhassan MM, Hemeg HA, Elmekki MA, Turkistani KA, AbdulAziz AA. Burden of Multidrug Resistant Mycobacterium tuberculosis Among New Cases in Al-Madinah Al-Munawarah, Saudi Arabia. Infect Disord Drug Targets. 2017;17(1):14-23. doi:10.2174/ 1871526517666161116104242

47. Ahmed-Abakur EH, Saad Alnour TM. Detection of multidrug resistant Mycobacterium tuberculosis in Tabuk, Saudi Arabia, using Genotype MTBDRplus. Int $J$ Mycobacteriol. 2019;8(1):25-28. doi:10.4103/ijmy.ijmy_29_19
Infection and Drug Resistance

\section{Publish your work in this journal}

Infection and Drug Resistance is an international, peer-reviewed openaccess journal that focuses on the optimal treatment of infection (bacterial, fungal and viral) and the development and institution of preventive strategies to minimize the development and spread of resistance. The journal is specifically concerned with the epidemiology of antibiotic resistance and the mechanisms of resistance development and diffusion in both hospitals and the community. The manuscript management system is completely online and includes a very quick and fair peerreview system, which is all easy to use. Visit http://www.dovepress.com/ testimonials.php to read real quotes from published authors. 